\title{
Editorial
}

\section{Stochastic Systems 2014}

\author{
Weihai Zhang, ${ }^{1}$ Xuejun Xie, ${ }^{2}$ Suiyang Khoo, ${ }^{3}$ Guangchen Wang, \\ Wuquan $\mathrm{Li}^{5}{ }^{5}$ and Ming $\mathrm{Gao}^{6}$ \\ ${ }^{1}$ College of Electrical Engineering and Automation, Shandong University of Science and Technology, Qingdao 266590, China \\ ${ }^{2}$ Institute of Automation, Qufu Normal University, Qufu 273165, China \\ ${ }^{3}$ School of Engineering, Deakin University, Victoria 3216, Australia \\ ${ }^{4}$ School of Control Science and Engineering, Shandong University, Jinan 250061, China \\ ${ }^{5}$ School of Mathematics and Information, Ludong University, Yantai 264025, China \\ ${ }^{6}$ College of Information and Control Engineering, China University of Petroleum (East China), Qingdao 266580, China
}

Correspondence should be addressed to Weihai Zhang; w_hzhang@163.com

Received 19 October 2014; Accepted 19 October 2014

Copyright (c) 2015 Weihai Zhang et al. This is an open access article distributed under the Creative Commons Attribution License, which permits unrestricted use, distribution, and reproduction in any medium, provided the original work is properly cited.

Stochastic systems captured by Itô differential equations and stochastic difference equations play a prominent role in modern control theory, which describe the systems disturbed by the randomness in the forms of Brownian motion and white noise. With the development of mathematical finance, network control, biology systems, and multiagent, many challenging stochastic-control problems are springing up, which need to be deeply investigated by means of more advanced theories and tools. To reflect the most recent advances in stochastic systems, we are determined to organize this special issue.

This special issue is focused on the stochastic-control systems and their applications to stability, control, filtering, communication, and fault detection. Topics covered in this issue include (i) stochastic modeling, stability, and stabilization analysis, (ii) stochastic robust/optimal/adaptive control, (iii) stochastic filtering and estimation, (iv) stochastic differential game, and (v) applications of stochastic-control theory to finance, economics, fault detection, and so forth. This special issue has received a total of 82 submitted papers with only 40 papers accepted.

There are 13 manuscripts on the subject "stochastic modeling, stability, and stabilization analysis." In the following, we give a brief summary. The paper entitled "Discrete-time indefinite stochastic linear quadratic optimal control with second moment constraints" by W. Zhang and G. Li studies stochastic LQ problem with constraints on the terminal state, where the weighting matrices in the cost functional are allowed to be indefinite. The problem of state-feedback stabilization for a class of stochastic nonlinear systems is investigated by $\mathrm{H}$. Wang et al. in the paper "Asymptotic stabilization by state feedback for a class of stochastic nonlinear systems with timevarying coefficients." G. Li and M. Chen investigate the stability and the stabilizability of delayed stochastic systems in "The stability and stabilization of stochastic delay-time systems." In "Integer-valued moving average models with structural changes," K. Yu et al. present a first order integer-valued moving average model which provides a flexible framework for modeling a wide range of dependence structures. In "Further results on dynamic additive Hazard rate model," Z. Zhang and L. Zhang study the dynamic additive hazard rate model and investigate its aging properties for different aging classes. C. $\mathrm{Li}$ and J. Duan in "Impact of correlated noises on additive dynamical systems" consider Fokker-Planck type equations under the fractional white noise measure. By means of Lyapunov functions, Doob's martingale inequality, and Borel-Cantelli lemma, W. Zhu et al. give some sufficient conditions for the exponential stability in the mean square of a class of stochastic systems in "Exponential stability of stochastic systems with delay and Poisson jumps." In "Stochastic resonance in neuronal network motifs with Ornstein-Uhlenbeck colored noise," X. Lou considers the effect of the Ornstein-Uhlenbeck colored noise on the stochastic resonance of the feed-forward-loop network motif. In "Input-to-state stability for a class of switched 
stochastic nonlinear systems by an improved average dwell time method," the input-to-state stability in the mean property of switched stochastic nonlinear systems is investigated by $\mathrm{R}$. Guo et al. In "Optimal dividend and capital injection strategies in the Cramér-Lundberg risk model," Y. Li and G. Liu maximize the discounted dividends payments minus the penalized discounted capital injections. In "Boundedness of stochastic delay differential systems with impulsive control and impulsive disturbance," L. Wang et al. derive several sufficient conditions which guarantee the $p$-moment boundedness of nonlinear impulsive stochastic delay differential systems by using the Lyapunov-Razumikhin method and stochastic analysis techniques. In the paper "Exponential stability of neutral stochastic functional differential equations with twotime-scale Markovian switching," J. Hu and Z. Xu develop exponential stability of neutral stochastic equations modeled by a continuous-time Markov chain which has a large state space. Y. Li and Y. Shen discuss the impact of stochastic noise and connection weight matrices uncertainty on global exponential stability of hybrid BAM neural networks with reaction diffusion terms. It is found that the perturbed hybrid BAM neural networks preserve global exponential stability if the intensities of both stochastic noise and the connection weight matrix uncertainty are smaller than the defined upper threshold.

There are 8 contributions closely related to controlled stochastic differential equations. In "Nonlinear stochastic $H_{\infty}$ control with Markov jumps and $(x, u, v)$-dependent noise: finite and infinite horizon cases," L. Sheng et al. investigate the $H_{\infty}$ control problem for nonlinear stochastic Markov jump systems with state, control, and external disturbance-dependent noise. In "The $H_{\infty}$ control for bilinear systems with Poisson jumps," R. Zhang et al. discuss the state feedback $H_{\infty}$ control problem for bilinear stochastic systems driven by both Brownian motion and Poisson jumps. S. Wang and $\mathrm{Z}$. Wu focus on optimal control derived by forward-backward regimeswitching systems with impulse controls in "Maximum principle for optimal control problems of forward-backward regime-switching systems involving impulse controls." Maximum principles and verification theorems for optimality are obtained and are used to solve an optimal investment and consumption problem with recursive utility. In "Mean-field backward stochastic evolution equations in Hilbert spaces and optimal control for BSPDEs," R. Xu and T. Wu investigate an optimal control problem of backward stochastic partial differential equations. Existence and uniqueness of mild solutions to mean-field backward stochastic evolution equations in Hilbert spaces are proved. In "Terminal-dependent statistical inference for the FBSDEs models," Y. Song works out a nonparameter method to estimate parameters of backward stochastic differential equations from noisy data and terminal conditions. In "Adaptive neural output feedback control of stochastic nonlinear systems with unmodeled dynamics," X. Xia and T. P. Zhang propose an adaptive neural output feedback control scheme for stochastic systems with unmodeled dynamics and unmeasured states. X. Dai et al. investigate robust stochastic mean-square exponential stabilization and robust $H_{\infty}$ control for stochastic partial differential time delay systems in "Robust $H_{\infty}$ control for linear stochastic partial differential systems with time delay." Based on the Lyapunov stability theory and stochastic analysis technique, G. Chen et al. establish both delay-independent and delaydependent dissipativity criteria for nonlinear stochastic delay systems in "Dissipative delay-feedback control for nonlinear stochastic systems with time-varying delay."

The subject on stochastic filtering and estimation has occupied 7 contributions. In "Parallel array bistable stochastic resonance system with independent input and its signal-tonoise ratio improvement," W. Li et al. discuss the design enhancement of the bistable stochastic resonance performance on sinusoidal signal and Gaussian white noise. A new pruning algorithm for Gaussian mixture PHD filter is proposed by X. Yan in the paper "Iterative mixture component pruning algorithm for Gaussian mixture PHD filter," where the pruning algorithm is based on maximizing the posterior probability density of the mixture weights. In "Covariancebased estimation from multisensor delayed measurements with random parameter matrices and correlated noises," R. Caballero-Águila et al. address the optimal least-square linear estimation problem for a class of discrete-time multisensor linear stochastic systems subject to randomly delayed measurements with different delay rates. In "Stochastic signal processing for sound environment system with decibel evaluation and energy observation," A. Ikuta and H. Orimoto propose a stochastic signal processing method to predict the output response probability distribution of complex sound environment systems. A fusion algorithm based on linear minimum mean-square error estimation is provided by X. Yuan et al. in "Performance analysis for distributed fusion with different dimensional data." In "Two identification methods for dualrate sampled-data nonlinear output-error systems," J. Chen and R. Ding present two methods for dual-rate sampleddata nonlinear output-error systems, which can estimate the unknown parameters directly. A particle filter based trackbefore-detect algorithm is proposed for the monopulse high pulse repetition frequency pulse Doppler radar by F. Cai et al. in "Dual-channel particle filter based track-before-detect for monopulse radar."

There are 4 papers that are concerned with stochastic differential games. In "Linear quadratic nonzero sum differential games with asymmetric information," D. Chang and H. Xiao consider an LQ nonzero sum stochastic differential game, where the information available to players is asymmetric. In "Algorithms to solve stochastic $\mathrm{H}_{2} / \mathrm{H}_{\infty}$ control with statedependent noise," several algorithms are proposed to solve $\mathrm{H}_{2} / \mathrm{H}_{\infty}$ control problems of stochastic systems by M. Gao et al. X. Chen and Q. Zhu use a maximum principle method to study a partial information nonzero sum differential game of backward stochastic differential equation with jumps in "Nonzero sum differential game of mean-field BSDEs with jumps under partial information." Here a feature is that both the game system and the performance functional are of mean-field type. Z. Wu and Q. Zhang's paper "Backward stochastic $\mathrm{H}_{2} / \mathrm{H}_{\infty}$ control: infinite horizon case" establishes a necessary and sufficient condition for the existence of $\mathrm{H}_{2} / \mathrm{H}_{\infty}$ control of infinite horizon backward stochastic differential equations. 
There are also 8 contributions on applications of stochastic control theory. X. Cao in "An upper bound of large deviations for capacities" obtains a type of large deviation principle under the sublinear expectation. Y.-G. Zhang et al. in "Moving state marine SINS initial alignment based on high degree CKF" propose a moving state marine initial alignment method for strap-down inertial navigation system. In "The Gerber-Shiu discounted penalty function of Sparre Andersen risk model with a constant dividend barrier," Y. Huang and W. Yu construct a new Sparre Andersen risk model with a constant dividend barrier and derive an integrodifferential equation of the Gerber-Shiu discounted penalty function. One paper entitled "A closed-form solution for robust portfolio selection with worst-case CVaR risk measure" by L. Tang and A. Ling considers a robust portfolio selection problem with WCCVaR constraint and the corresponding closedform solution is obtained. In "Stochastic dominance under the nonlinear expected utilities," X. Xiao proposes a definition of stochastic dominance under nonlinear expected utilities and gives sufficient conditions on which a random choice $X$ stochastically dominates a random choice $Y$ under the nonlinear expected utilities. In "Equilibrium model of discrete dynamic supply chain network with random demand and advertisement strategy," G. Zhang et al. analyze the impact of advertising investment on a discrete dynamic supply chain network which consists of suppliers, manufactures, retailers, and demand markets associated at different tiers under random demand. In "Research on multiprincipals selecting effective agency mode in the student loan system," agency modes are discussed by building different principal agent models to solve incentive problems in student loan system. In the paper " $O n H_{\infty}$ fault estimator design for linear discrete time-varying systems under unreliable communication link," $\mathrm{Y}$. Li et al. investigate the $H_{\infty}$ fixed-lag fault estimator design for linear discrete time-varying systems with intermittent measurements, which is described by a Bernoulli distributed random variable.

\title{
Acknowledgments
}

This special issue represents an exciting and insightful snapshot of the current stochastic systems research. As the Lead Guest Editor of this special issue, I would like to express my sincere gratitude to my five coeditors for helping me to undertake this project with a wonderful accomplishment. We are also deeply appreciative of interested authors who have submitted their papers to the special issue.

\author{
Weihai Zhang \\ Xuejun Xie \\ Suiyang Khoo \\ Guangchen Wang \\ Wuquan Li \\ Ming Gao
}




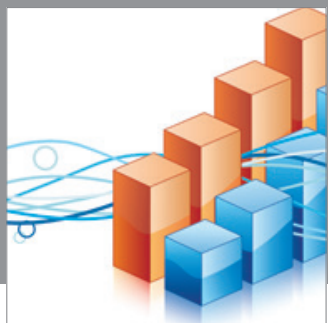

Advances in

Operations Research

mansans

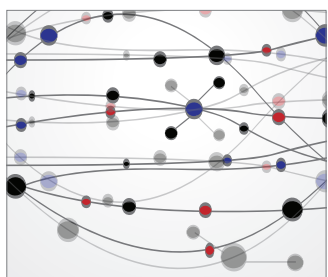

The Scientific World Journal
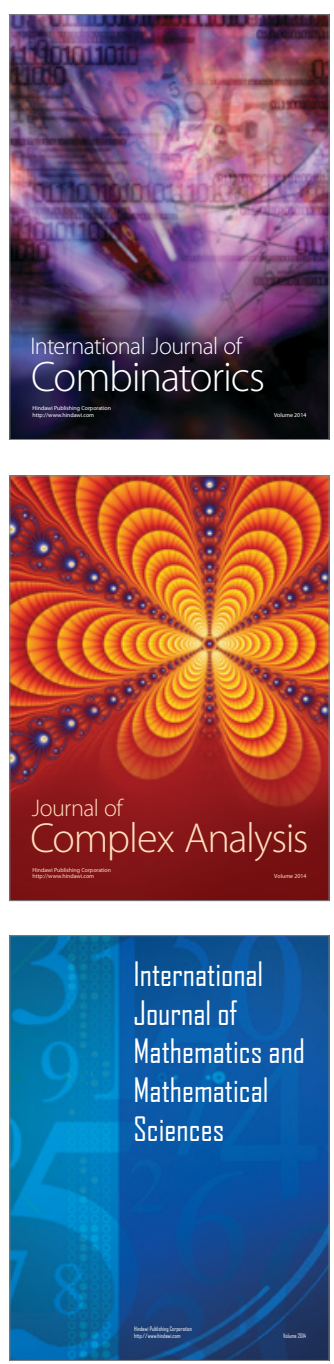
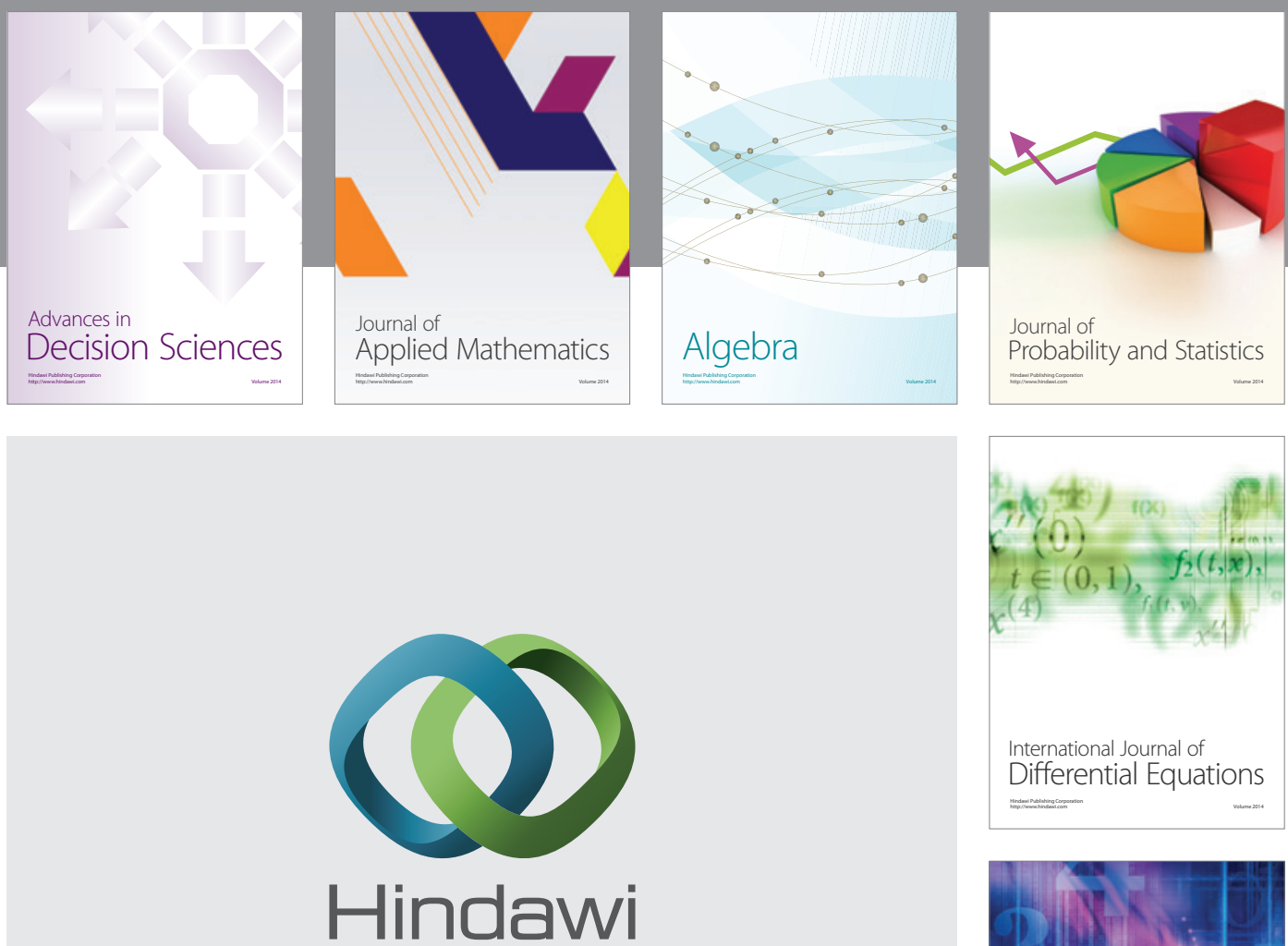

Submit your manuscripts at http://www.hindawi.com
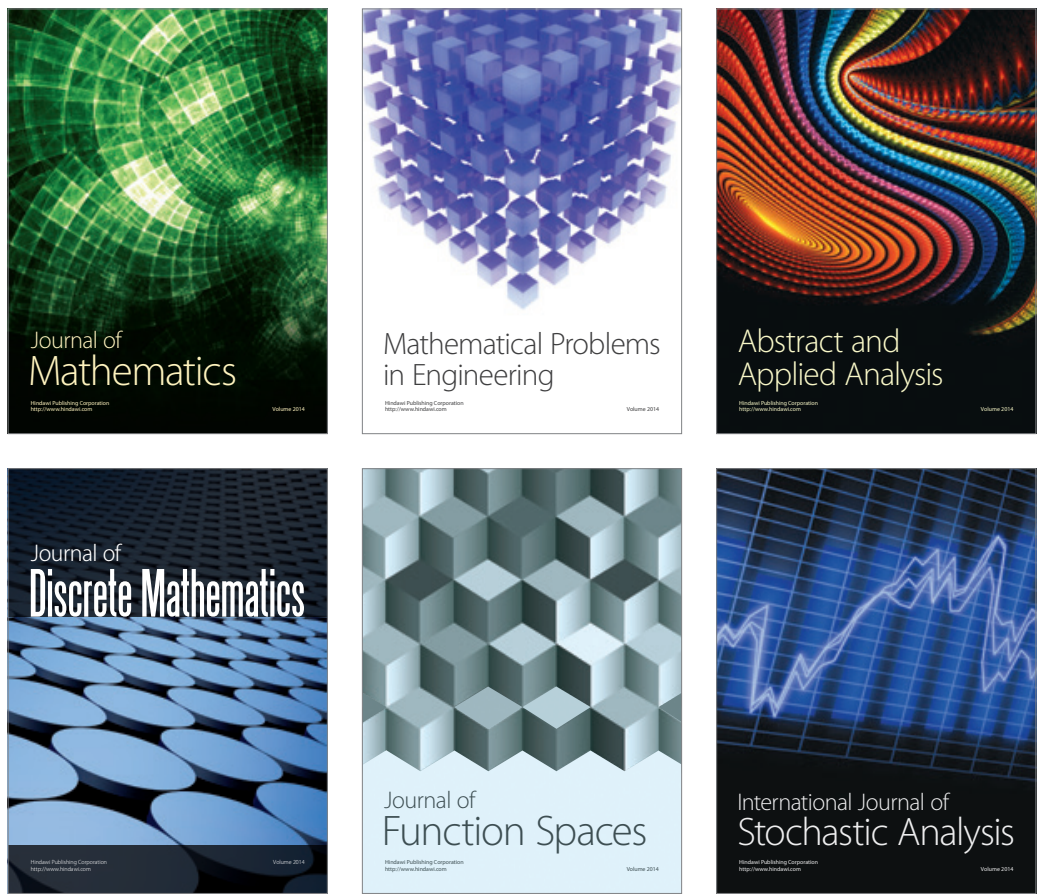

Journal of

Function Spaces

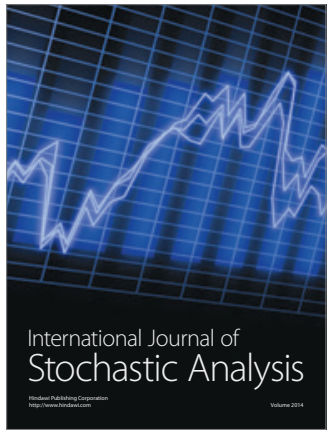

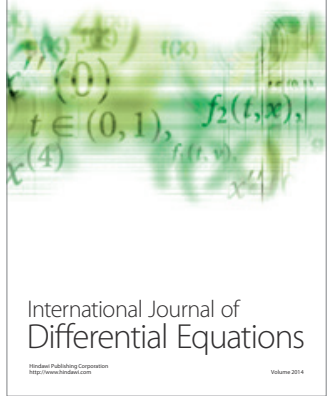
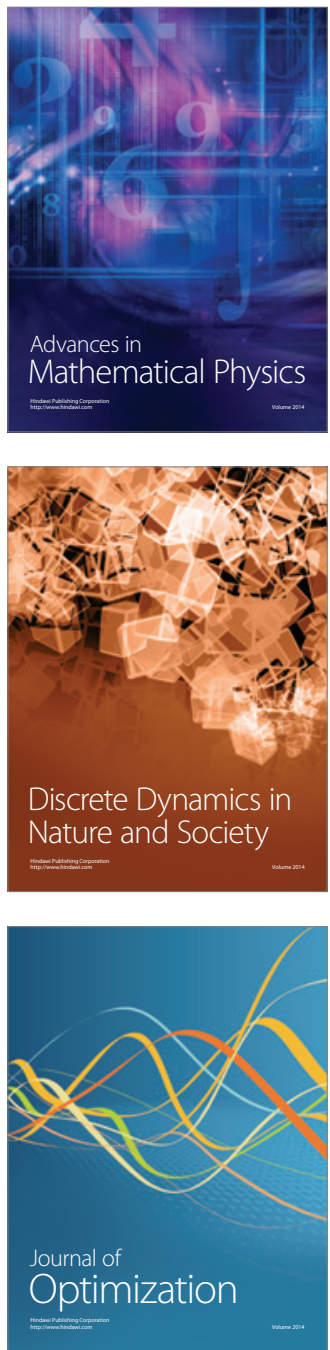